TRANSACTIONS OF THE

AMERICAN MATHEMATICAL SOCIETY

Volume 360, Number 6, June 2008, Pages 3193-3210

S 0002-9947(08)04395-X

Article electronically published on January 10, 2008

\title{
ON INITIAL SEGMENT COMPLEXITY AND DEGREES OF RANDOMNESS
}

\author{
JOSEPH S. MILLER AND LIANG YU
}

\begin{abstract}
One approach to understanding the fine structure of initial segment complexity was introduced by Downey, Hirschfeldt and LaForte. They define $X \leq_{K} Y$ to mean that $(\forall n) K(X \mid n) \leq K(Y \nmid n)+O(1)$. The equivalence classes under this relation are the $K$-degrees. We prove that if $X \oplus Y$ is 1-random, then $X$ and $Y$ have no upper bound in the $K$-degrees (hence, no join). We also prove that $n$-randomness is closed upward in the $K$-degrees. Our main tool is another structure intended to measure the degree of randomness of real numbers: the $v L$-degrees. Unlike the $K$-degrees, many basic properties of the $v L$-degrees are easy to prove. We show that $X \leq_{K} Y$ implies $X \leq_{v L} Y$, so some results can be transferred. The reverse implication is proved to fail. The same analysis is also done for $\leq_{C}$, the analogue of $\leq_{K}$ for plain Kolmogorov complexity.

Two other interesting results are included. First, we prove that for any $Z \in 2^{\omega}$, a 1-random real computable from a $1-Z$-random real is automatically $1-Z$-random. Second, we give a plain Kolmogorov complexity characterization of 1-randomness. This characterization is related to our proof that $X \leq_{C} Y$ implies $X \leq_{v L} Y$.
\end{abstract}

\section{INTRODUCTION}

This paper is part of an ongoing project to understand the initial segment complexity of random real numbers (by which we mean elements of $2^{\omega}$ ). Several authors have investigated oscillations in the complexity of initial segments of 1-random (i.e., Martin-Löf random) reals, with respect to either plain or prefix-free Kolmogorov complexity (denoted by $C$ and $K$, respectively). These include Martin-Löf $[19,20]$, Chaitin [1, 3], Solovay [29] and van Lambalgen [30].

Our approach is different. While previous work focuses on describing the behavior of the initial segment complexity of a real number, we instead focus on interpreting that behavior. We argue that the initial segment complexity of $X \in 2^{\omega}$ carries useful information about $X$. An obvious example is Schnorr's theorem that $X \in 2^{\omega}$ is 1 -random iff $(\forall n) K(X\lceil n) \geq n-O(1)$. A more recent example is the fact that $X \in 2^{\omega}$ is 2-random iff $\left(\exists^{\infty} n\right) C(X \uparrow n) \geq n-O(1)$ (see Miller [21]; Nies,

Received by the editors May 30, 2006.

2000 Mathematics Subject Classification. Primary 68Q30, 03D30, 03D28.

The first author was partially supported by the Marsden Fund of New Zealand and by an NSF VIGRE postdoctoral fellowship at Indiana University.

The second author was supported by a postdoctoral fellowship from the New Zealand Institute for Mathematics and its Applications, NSF of China No.10471060 and No.10420130638, and by No.R-146-000-054-123 of Singapore: Computability Theory and Algorithmic Randomness. Both authors were supported by the Institute for Mathematical Sciences, National University of Singapore, during the Computational Aspects of Infinity program in 2005.

(C) 2008 American Mathematical Society 3193

Reverts to public domain 28 years from publication 
Stephan and Terwijn [24]). These results raise obvious questions: can 1-randomness be characterized in terms of initial segment $C$-complexity - a long elusive goalor 2-randomness in terms of initial segment $K$-complexity? We will give positive answers to both questions.

Many of our results will be stated in terms of the $K$-degrees, which were introduced by Downey, Hirschfeldt and LaForte [6, 7]. Write $X \leq_{K} Y$ if $Y$ has higher initial segment prefix-free complexity than $X$ (up to a constant). Formally, $(\forall n) K(X\lceil n) \leq K(Y\lceil n)+O(1)$. The induced partial order is called the $K$ degrees. Define the $C$-degrees in the same way. This brings us to the second major theme of this paper: degrees of randomness. What does it means to say that one real number is more random than another? Based on the intuition that higher complexity implies more randomness, one might think that $X \leq_{K} Y$ (or $X \leq_{C} Y$ ) means that $Y$ is more random than $X$. We will provide some evidence supporting this view.

Our effort to connect the properties of a real number to its initial segment complexity culminates in Corollary 7.5, which states that $X \oplus Z$ is 1-random iff

$$
(\forall n) C(X\lceil n)+K(Z\lceil n) \geq 2 n-O(1) .
$$

Thus, the initial segment $C$-complexity of $X \in 2^{\omega}$ gives a complete accounting of the reals $Z \in 2^{\omega}$ such that $X \oplus Z$ is 1-random. By symmetry, the same information is implicit in the initial segment $K$-complexity of $X$.

We will see that the corollary says more, but first we introduce the $v L$-degrees. These are a slight variant of the $L R$-degrees, which were introduced by Nies [23]; see Section 3 for details. Write $X \leq_{v L} Y$ if $\left(\forall Z \in 2^{\omega}\right)[X \oplus Z$ is 1-random $\Longrightarrow$ $Y \oplus Z$ is 1-random]. The induced partial order is called the van Lambalgen degrees (or $v L$-degrees) because the definition was motivated by a theorem of van Lambalgen (Theorem 3.1). These degrees offer an alternative way to gauge randomness, one based on the global properties of reals, not on their finite initial segments. Corollary 7.5 shows that $X \leq_{C} Y$ implies $X \leq_{v L} Y$, and again by symmetry, that $X \leq_{K} Y$ implies $X \leq_{v L} Y$. Because many properties of the $v L$-degrees are easily proved, this new structure is a useful tool in studying the $K$-degrees and $C$-degrees. For example, we will show that if $X \leq_{v L} Y$ and $X$ is $n$-random (i.e., 1-random relative to $\left.\emptyset^{(n-1)}\right)$, then $Y$ is also $n$-random. By the above implications, every real with higher initial segment complexity than an $n$-random real must also be $n$ random. As promised, this supports the assertion that reals with higher $K$-degree (or $C$-degree) are more random.

The article is organized as follows. Section 2 covers the necessary concepts from Kolmogorov complexity and Martin-Löf randomness. The van Lambalgen degrees are introduced in Section 3 and several basic properties are proved. Section 4 is a digression from the main topics of the paper; in it we prove that any 1-random real computable from a $1-Z$-random real is automatically $1-Z$-random. This follows easily from van Lambalgen's theorem if $Z \in 2^{\omega}$ has 1-random Turing degree, but the general case requires more work. In Section 5, we prove that $X \leq_{K} Y$ implies $X \leq_{v L} Y$ and derive several results about the $K$-degrees. As was observed above, this result follows from Corollary 7.5. But it is also an immediate consequence of Theorem 5.1, which in turn is used in the proof of Corollary 7.5. Section 6 offers three results that contrast the $K$-degrees and the $v L$-degrees. In particular, Proposition 6.2 shows that 1 -random reals that differ by a computable permutation need not be $K$-equivalent (although they must be $v L$-equivalent), which demonstrates the 
essentially "local" nature of the $K$-degrees. The final section focuses on plain complexity. We prove that $X \in 2^{\omega}$ is 1-random iff $(\forall n) C(X\lceil n) \geq n-K(n)-O(1)$. Alternatively, $X$ is 1-random iff for every computable $g: \omega \rightarrow \omega$ such that $\sum_{n \in \omega} 2^{-g(n)}$ is finite, $(\forall n) C(X\lceil n) \geq n-g(n)-O(1)$. Finally, we prove Corollary 7.5 and derive several consequences for the $C$-degrees.

We finish this section with a brief discussion of how our results fit in with the previous work on the $K$-degrees of 1 -random reals. It follows from work of Solovay [29] that Chaitin's halting probability $\Omega$ has a different $K$-degree than any arithmetically random real. Hence, there are at least two $K$-degrees. Yu, Ding and Downey [33] proved that $\mu\left\{X \in 2^{\omega}: X \leq_{K} Y\right\}=0$, for any $Y \in 2^{\omega}$. From this, they conclude that there are uncountably many 1-random $K$-degrees (an explicit construction of an antichain of size $2^{\aleph_{0}}$ is given in [32]). An early goal of the present research was to calculate the measure of $\left\{Y \in 2^{\omega}: X \leq_{K} Y\right\}$. It must be noted that this measure depends on the choice of $X \in 2^{\omega}$. If $X$ is computable, then it is $K$-below every real; hence $\mu\left\{Y \in 2^{\omega}: X \leq_{K} Y\right\}=1$. On the other hand, the result of $\mathrm{Yu}$, Ding and Downey implies that $\mu\left\{X \oplus Y \in 2^{\omega}: X \leq_{K} Y\right\}=0$. Hence, $\mu\left\{Y \in 2^{\omega}: X \leq_{K} Y\right\}=0$ for almost all $X \in 2^{\omega}$. Now by an easy complexity calculation, the measure is zero when $X$ is (weakly) 2-random. In fact, it follows from Corollary 5.3 (ii) that 1-randomness is sufficient. It should be noted that this condition does not characterize 1-randomness; it is easy to construct a non-1-random real $X \in 2^{\omega}$ for which $\mu\left\{Y \in 2^{\omega}: X \leq_{K} Y\right\}=0$.

Several results in this paper produce incomparable 1 -random $K$-degrees, but none prove the existence of comparable 1 -random $K$-degrees. That is done in a companion paper [22], where we prove that for any 1-random $Y \in 2^{\omega}$, there is a 1-random $X \in 2^{\omega}$ such that $X<_{K} Y$ (in fact, $\lim _{n \rightarrow \infty} K(Y \uparrow n)-K(X\lceil n)=\infty$ ). Another problem that is not addressed in this paper is whether the $C$-degrees and the $K$-degrees differ for 1-random reals. They are known to be different in general $[29,7]$, but their relationship on the 1-random reals remains entirely open.

\section{Preliminaries}

We begin with a brief review of effective randomness and Kolmogorov complexity. A more complete introduction can be found in Li and Vitányi [18] or the upcoming monograph of Downey and Hirschfeldt [5]. We assume that the reader is familiar with the basics of computability theory and measure theory. Soare [27] and Oxtoby [25] are good resources for these subjects.

Martin-Löf [19] introduced the most successful notion of effective randomness for real numbers. A Martin-Löf test is a uniform sequence $\left\{\mathcal{G}_{n}\right\}_{n \in \omega}$ of $\Sigma_{1}^{0}$ classes such that $\mu\left(\mathcal{G}_{n}\right) \leq 2^{-n}$. A real $X \in 2^{\omega}$ is said to pass a Martin-Löf test $\left\{\mathcal{G}_{n}\right\}_{n \in \omega}$ if $X \notin \bigcap_{n \in \omega} \mathcal{G}_{n}$. We say that $X \in 2^{\omega}$ is 1-random (or Martin-Löf random) if it passes all Martin-Löf tests. This notion generalizes naturally; for any $n \in \omega$ and oracle $Z \in 2^{\omega}$, define $n$ - $Z$-randomness by replacing the $\Sigma_{1}^{0}$ classes with $\Sigma_{n}^{0}[Z]$ classes in Martin-Löf's definition. The two parameters are related by the jump operator:

Theorem 2.1 (Kurtz [14]). For $n \in \omega$ and $Z \in 2^{\omega}$, $n$-Z-randomness is equivalent to $1-Z^{(n-1)}$-randomness.

We write $n$-random for $n$ - $\emptyset$-random, or equivalently, $1-\emptyset^{(n-1)}$-random.

Kolmogorov [11] and Solomonoff [28] defined the complexity of a finite string to be the length of its shortest description. Formally, we use a partial computable 
function $M: 2^{<\omega} \rightarrow 2^{<\omega}$ to "decode" descriptions. Then the Kolmogorov complexity of $\sigma \in 2^{<\omega}$ with respect to $M$ is $C_{M}(\sigma)=\min \{|\tau|: M(\tau)=\sigma\}$. There is an essentially optimal choice for the decoding function: a partial computable $V: 2^{<\omega} \rightarrow 2^{<\omega}$ with the property that if $M: 2^{<\omega} \rightarrow 2^{<\omega}$ is any other partial computable function, then $\left(\forall \sigma \in 2^{<\omega}\right) C_{V}(\sigma) \leq C_{M}(\sigma)+O(1)$. We call $V$ the universal machine and call $C(\sigma)=C_{V}(\sigma)$ the plain (Kolmogorov) complexity of $\sigma \in 2^{<\omega}$.

Levin [16] and Chaitin [2] introduced a modified form of Kolmogorov complexity that has natural connections to the Martin-Löf definition of randomness. For finite binary strings $\sigma, \tau \in 2^{<\omega}$, we write $\sigma \prec \tau$ to mean that $\sigma$ is a proper prefix of $\tau$. Similarly, $\sigma \prec X$ means that $\sigma$ is an initial segment of $X \in 2^{\omega}$. A set of strings $D \subseteq 2^{<\omega}$ is prefix-free if $(\forall \sigma, \tau \in D) \sigma \nprec \tau$. A partial function $M: 2^{<\omega} \rightarrow 2^{<\omega}$ is prefix-free if its domain is a prefix-free set. If $M$ is prefix-free, then we write $K_{M}$ instead of $C_{M}$ for the Kolmogorov complexity with respect to $M$. As before, there is a universal prefix-free machine $U: 2^{<\omega} \rightarrow 2^{<\omega}$ that is optimal for prefix-free partial computable functions, in the sense that $\left(\forall \sigma \in 2^{<\omega}\right) K_{U}(\sigma) \leq K_{M}(\sigma)+O(1)$, for any such function $M$. We write $K(\sigma)$ for $K_{U}(\sigma)$ and call it the prefix-free complexity of $\sigma \in 2^{<\omega}$.

It is well known that the 1-random reals can be characterized in terms of the prefix-free complexity of their initial segments.

Theorem 2.2 (Schnorr). $X \in 2^{\omega}$ is 1-random iff $(\forall n) K(X\lceil n) \geq n-O(1)$.

Theorem 7.1 gives a similar characterization in terms of plain complexity.

We now review some of the combinatorics of prefix-free complexity. The fact that $U$ has prefix-free domain implies that $\sum_{\sigma \in 2<\omega} 2^{-K(\sigma)} \leq 1$; this is Kraft's inequality. It is clear that $\left(\forall \sigma \in 2^{<\omega}\right) C(\sigma) \leq|\sigma|+O(1)$, but this would clearly violate Kraft's inequality were it true of prefix-free complexity. Instead, the natural upper bound on $K$ is given by the following result.

Lemma 2.3 (Chaitin [2]). $\quad$ (i) $\left(\forall \sigma \in 2^{<\omega}\right) K(\sigma) \leq|\sigma|+K(|\sigma|)+O(1)$.

(ii) $(\forall n)(\forall k)\left|\left\{\sigma \in 2^{n}: K(\sigma) \leq n+K(n)-k\right\}\right| \leq 2^{n-k+O(1)}$.

Observe that $K$ is applied to natural numbers as well as to binary strings. This is possible because we identify finite binary strings with natural numbers. In particular, $\sigma \in 2^{<\omega}$ represents $n \in \omega$ if the binary expansion of $n+1$ is $1 \sigma$. Note that strings of length $n$ are identified with numbers between $2^{n}-1$ and $2^{n+1}-2$. Having fixed a natural effective bijection between $2^{<\omega}$ and $\omega$, we may view $K$ as a function of $\omega$ when it is convenient.

Information content measures provide an alternative approach to prefix-free complexity. These were introduced by Levin and Zvonkin [34] and studied further by Levin $[17,16]$. They are implicit in Chaitin [2] and the name comes from his later paper [3]. A function $\widehat{K}: \omega \rightarrow \omega \cup\{\infty\}$ is an information content measure if

(i) $\sum_{n \in \omega} 2^{-\widehat{K}(n)}$ converges (where $2^{-\infty}=0$ ).

(ii) $\{\langle n, k\rangle: \widehat{K}(n) \leq k\}$ is computable enumerable.

Not only is $K$ an information content measure (when viewed as a function of $\omega$ ), but it is minimal [16]: if $\widehat{K}$ is another information content measure, then $(\forall n) K(n) \leq$ $\widehat{K}(n)+O(1)$.

We write $C^{Z}$ and $K^{Z}$ for the relativizations of plain and prefix-free complexity to an oracle $Z \in 2^{\omega}$. The results mentioned above remain true in their relativized forms. In particular, $X \in 2^{\omega}$ is 1 - $Z$-random iff $(\forall n) K^{Z}(X\lceil n) \geq n-O(1)$. The 
following result relates $K^{Z}$ to unrelativized prefix-free complexity when $Z \in 2^{\omega}$ is 1-random.

Ample Excess Lemma. Let $Z \in 2^{\omega}$ be 1-random.

(i) $\sum_{n \in \omega} 2^{n-K(Z \nmid n)}<\infty$.

(ii) $(\forall n) K^{Z}(n) \leq K(Z\lceil n)-n+O(1)$.

Proof. (i) Note that, for any $m \in \omega$,

$$
\begin{aligned}
& \sum_{\sigma \in 2^{m}} \sum_{n \leq m} 2^{n-K(\sigma \mid n)}=\sum_{\sigma \in 2^{m}} \sum_{\tau \prec \sigma} 2^{|\tau|-K(\tau)} \\
&=\sum_{\tau \in 2 \leq m} 2^{m-|\tau|} 2^{|\tau|-K(\tau)}=2^{m} \sum_{\tau \in 2 \leq m} 2^{-K(\tau)} \leq 2^{m},
\end{aligned}
$$

where the last step is Kraft's inequality. Therefore, for any $p \in \omega$, there are at most $2^{m} / p$ strings $\sigma \in 2^{m}$ for which $\sum_{n \leq m} 2^{n-K(\sigma \mid n)}>p$. This implies that $\mathcal{G}_{p}=\left\{X \in 2^{\omega}: \sum_{n \in \omega} 2^{n-K(X \uparrow n)}>p\right\}$ has measure at most $1 / p$. Thus $\left\{\mathcal{G}_{2^{k}}\right\}_{k \in \omega}$ clearly a uniform sequence of $\Sigma_{1}^{0}$ classes - is a Martin-Löf test. Therefore, $Z \notin \mathcal{G}_{2^{k}}$ for some $k \in \omega$, and so $\sum_{n \in \omega} 2^{n-K(Z \nmid n)} \leq 2^{k}$.

(ii) Define $\widehat{K}(n)=K(Z\lceil n)-n$. Note that $\{\langle n, k\rangle: \widehat{K}(n) \leq k\}$ is computably enumerable from $Z$. Hence by (i), $\widehat{K}$ is an information content measure relative to $Z$. The result now follows from the minimality of $K^{Z}$ among such functions.

\section{The Van Lambalgen Degrees}

When is a given 1-random real more random than another? The $K$-degrees attempt to answer this question using initial segment complexity. In this section, we propose a different approach - one based on the global behavior of real numbers, rather than their local structure. Our definition will be motivated by the following result.

Theorem 3.1 (van Lambalgen [31]). If $X, Y \in 2^{\omega}$, then $X \oplus Y$ is 1-random iff $X$ is 1-random and $Y$ is 1 - $X$-random.

Nies [23] defined $X \geq_{L R} Y$ to mean

$$
\left(\forall Z \in 2^{\omega}\right)[Z \text { is } 1 \text { - } X \text {-random } \Longrightarrow Z \text { is } 1-Y \text {-random }] \text {. }
$$

By Theorem 3.1, if $X$ and $Y$ are both 1-random, then $X \geq_{L R} Y$ iff $X \oplus Z$ is 1-random implies that $Y \oplus Z$ is 1-random, for all $Z \in 2^{\omega}$. Taking this partial characterization of $\geq_{L R}$ as a definition, we write $X \leq_{v L} Y$ iff

$$
\left(\forall Z \in 2^{\omega}\right)[X \oplus Z \text { is 1-random } \Longrightarrow Y \oplus Z \text { is 1-random }] .
$$

We call the equivalence classes induced by this relation the van Lambalgen degrees.

The $v L$-degrees differ from the $L R$-degrees in two relatively minor ways: the least $v L$-degree contains exactly the non-1-random reals (part (ii) of Theorem 3.4), and for 1-random reals, $\leq_{v L}$ is equivalent to $\geq_{L R}$. Both changes make $\leq_{v L}$ more plausible as a measure of relative randomness. For the first, note that $X \equiv_{T} Y$ implies $X \equiv_{L R} Y$, so every $L R$-degree contains non-1-random reals. On the other hand, the $v L$-degree of a 1-random real contains only 1-random reals. But why the reversal of the ordering on 1-random reals? We will see in Corollaries 5.2 and 7.6 that both $\leq_{K}$ and $\leq_{C}$ imply $\leq_{v L}$. So the direction of the ordering is appropriate 
for our purposes and $X \leq_{v L} Y$ can reasonably be interpreted as saying that $Y$ is more random than $X$.

The fact that both initial segment notions refine the $v L$-degrees allows us to transfer facts about the $v L$-degrees to these other structures, which is useful because many basic properties of the $v L$-degrees are easy to prove from known results. In addition to Theorem 3.1, we will use the following two facts. The first is closely related to a result proved independently by Gács [10]: every real is computable from a 1-random real.

Theorem 3.2 (Kučera [12]). There is a 1-random real in every Turing degree $\geq \mathbf{0}^{\prime}$.

Theorem 3.3 (Kučera and Terwijn [13]). For every $X \in 2^{\omega}$, there is a $W \varliminf_{T} X$ so that every 1 - $X$-random real is $1-X \oplus W$-random.

Theorem 3.4 (Basic properties of the $v L$-degrees).

(i) If $X \leq_{v L} Y$ and $X$ is $n$-random, then $Y$ is n-random.

(ii) The least $v L$-degree is $\mathbf{0}_{v L}=\left\{X \in 2^{\omega}: X\right.$ is not 1-random $\}$.

(iii) If $X \oplus Y$ is 1-random, then $X$ and $Y$ have no upper bound in the $v L$-degrees.

(iv) If $Y \leq_{T} X$ and $Y$ is 1-random, then $X \leq_{v L} Y$.

(v) There are 1 -random reals $X \equiv_{v L} Y$ but $X<_{T} Y$.

Proof. (i) Assume that $X \leq_{v L} Y$ and $X$ is $n$-random. First consider $n=1$. Select a 1 - $X$-random real $Z$. Then $X \oplus Z$ is 1-random, so $Y \oplus Z$ is 1 -random. Thus $Y$ is 1 -random.

Now take $n>1$. By Theorem 3.2, there is a 1-random real $Z \equiv_{T} \emptyset^{(n-1)}$. Then $X$ is $n$-random $\Longrightarrow X$ is 1 - $Z$-random $\Longrightarrow X \oplus Z$ is 1 -random $\Longrightarrow Y \oplus Z$ is 1 -random $\Longrightarrow Y$ is 1 - $Z$-random $\Longrightarrow Y$ is $n$-random.

(ii) Clearly, if $X$ is not 1-random, then $X \leq_{v L} Y$ for any real $Y$. If $X$ is 1random, then $X \Varangle_{v L} \emptyset$, or else $\emptyset$ would be 1 -random by part (i). Therefore, $\mathbf{0}_{v L}$ consists exactly of the non-1-random reals.

(iii) Let $X \oplus Y$ be 1-random and assume, for a contradiction, that $X, Y \leq{ }_{v L} Z$. Because $X \leq_{v L} Z$ and $X \oplus Y$ is 1-random, $Z \oplus Y$ is 1-random too. Therefore, $Y \oplus Z$ is 1-random. But $Y \leq_{v L} Z$, so $Z \oplus Z$ must also be 1 -random, which is a contradiction.

(iv) Assume that $Y \leq_{T} X$ and $Y$ is 1-random. For any $Z \in 2^{\omega}, X \oplus Z$ is 1-random $\Longrightarrow Z$ is 1 - $X$-random $\Longrightarrow Z$ is $1-Y$-random $\Longrightarrow Y \oplus Z$ is 1-random. Therefore, $X \leq_{v L} Y$.

(v) Pick any 1-random real $X \geq_{T} \emptyset^{\prime}$ (e.g., the halting probability $\Omega$; see below). By Theorem 3.3, there is a $W \not_{T} X$ such that every 1 - $X$-random real is $1-X \oplus W$ random. Take a 1-random real $Y \equiv_{T} X \oplus W$; this is guaranteed to exist by Theorem 3.2. So $X<_{T} Y$. Also, $Z \oplus X$ is 1-random $\Longleftrightarrow Z$ is $1-X$-random $\Longleftrightarrow$ $Z$ is $1-X \oplus W$-random $\Longleftrightarrow Z$ is $1-Y$-random $\Longleftrightarrow Z \oplus Y$ is 1 -random. Therefore, $X \equiv_{v L} Y$.

Corollary 3.5 (More basic properties of the $v L$-degrees).

(i) There is no join in the vL-degrees.

(ii) If $X \oplus Y$ is 1-random, then $X \oplus Y<_{v L} X, Y$ and $\left.X\right|_{v L} Y$.

(iii) There is no maximal $v L$-degree.

\footnotetext{
${ }^{1}$ Nies [23] observed that $Y \leq_{T} X$ implies $Y \leq_{L R} X$, from which part (iv) of Theorem 3.4 follows.
} 
(iv) There is no minimal 1-random vL-degree.

(v) Every finite poset can be embedded into $\left(2^{\omega}, \leq_{v L}\right)$.

Proof. (i) Immediate from part (iii) of Theorem 3.4.

(ii) By part (iii) of Theorem 3.4, X $\left.\right|_{v L} Y$. By part (iv) of the same theorem, $X \oplus Y \leq_{v L} X, Y$. Therefore, $X \oplus Y<_{v L} X, Y$.

(iii) If $X=X_{1} \oplus X_{2}$ is 1-random, then $X<_{v L} X_{1}, X_{2}$ by part (ii). So the $v L$-degree of $X$ is not maximal.

(iv) Assume that $X$ is 1-random. Take any 1 - $X$-random real $Y$; thus $X \oplus Y$ is 1-random. By part (ii), $\emptyset<_{v L} X \oplus Y<_{v L} X$. So, there is no minimal 1-random $v L$-degree.

(v) Suppose that $\mathbb{P}=(P, \leq)$ is a finite partial order; let $P=\left\{p_{i}\right\}_{i<n}$. Pick a 1-random real $X=\bigoplus_{i<n} X_{i}$. For any $k<n$, define $F(k)=\left\{i: p_{k} \leq p_{i}\right\}$ and let $Y_{k}=\bigoplus_{i \in F(k)} X_{i}$. Let $g: P \rightarrow 2^{\omega}$ be defined by $g\left(p_{k}\right)=Y_{k}$. It suffices to prove that $p_{j} \leq p_{k} \Longleftrightarrow Y_{j} \leq_{v L} Y_{k}$. If $p_{j} \leq p_{k}$, then $F(k) \subseteq F(j)$ so $Y_{j} \leq_{v L} Y_{k}$, by part (i). If $p_{j} \not \leq p_{k}$, then $k \notin F(j)$ and so $Y_{j} \oplus X_{k}$ is 1-random. But $Y_{k} \oplus X_{k}$ is not 1-random since $k \in F(k)$. So $Y_{j} \not \mathbb{\leq}_{v L} Y_{k}$.

Note that part $(\mathrm{v})$ of the corollary implies that the $\Sigma_{1}^{0}$ theory of $\left(2^{\omega}, \leq_{v L}\right)$ is decidable, as in Lerman [15].

We finish the section by considering the $v L$-degrees of specific reals. Chaitin [2] proposed the halting probability $\Omega$ of the universal prefix-free machine $U$ as a natural example of a 1-random real. Formally, let $\Omega=\sum_{U(\sigma) \downarrow} 2^{-|\sigma|}$. It is known that $\Omega \equiv_{T} \emptyset^{\prime}$. The construction of $\Omega$ has a natural relativization. For any oracle $Z \in 2^{\omega}$, let $U^{Z}$ be universal for prefix-free machines relative to $Z$. Let $\Omega^{Z}=\sum_{U^{Z}(\sigma) \downarrow} 2^{-|\sigma|}$. Relativizing the usual proofs, $\Omega^{Z}$ is 1 - $Z$-random and computable from $Z^{\prime}$. Using the results above, there are several simple observations we can make about the $v L$-degrees of $\Omega$, the columns of $\Omega$, and $\Omega^{\emptyset^{(n)}}$ for $n \in \omega$.

First, it is worth asking if the $v L$-degree of $\Omega^{Z}$ is independent of the choice of $U^{Z}$. It is not. The $v L$-degree of $\Omega$ is well defined, but this is not even true for $\Omega^{\emptyset^{\prime}}$. It can be proved (using ideas in [8]) that if $Z \in 2^{\omega}$ has 1-random degree, then different choices of $U^{Z}$ can give values of $\Omega^{Z}$ that are 1-random relative to each other. Therefore, by Theorem 3.4 (iii), different versions of $\Omega^{Z}$ have different $v L$-degrees. Even for a uniform choice of universal machines, there must be oracles $Z_{0}, Z_{1} \in 2^{\omega}$ that differ by only a finite amount but for which $\Omega^{Z_{1}}$ and $\Omega^{Z_{2}}$ are relatively random [8], hence have no upper bound in the $v L$-degrees. Despite these issues, the results below depend only on the most elementary properties of $\Omega^{Z}$ and are independent of the choices of universal machines.

Corollary 3.6 (The $v L$-degrees of distinguished reals).

(i) $\Omega$ is the least $\Delta_{2}^{0} 1$-random real in the vL-degrees.

(ii) For $n>1$, an $n$-random and a $\Delta_{n}^{0} 1$-random have no $\leq_{v L}$ upper bound.

(iii) If $m \neq n$, then $\Omega^{\emptyset^{(n)}}$ and $\Omega^{\emptyset^{(m)}}$ have no upper bound in the vL-degrees.

(iv) If $\Omega=\bigoplus_{n \in \omega} \Omega_{n}$, then $\left\{\Omega_{n}\right\}_{n \in \omega}$ is a vL-antichain of $\Delta_{2}^{0} 1$-random reals (and again, no two have an upper bound in the vL-degrees).

Proof. (i) follows from part (iv) of Theorem 3.4 and the fact that $\Omega \equiv_{T} \emptyset^{\prime}$. For (ii), assume that $X \in 2^{\omega}$ is a $\Delta_{n}^{0} 1$-random real. If $Y \in 2^{\omega}$ is $n$-random, then $Y$ is 1 - $X$-random. Now apply Theorem 3.4 (iii). In (iii), we can assume that $m>n$. 
Note that $\Omega^{\emptyset^{(n)}}$ is a $\Delta_{n+1}^{0} 1$-random real and that $\Omega^{\emptyset^{(m)}}$ is $(n+1)$-random. So, (ii) implies (iii). Finally, (iv) follows from Theorem 3.4 (iii).

\section{Digression: The Turing Degrees of $1-Z$-RAndom ReAls}

The point of departure for this section is the observation that Theorem 3.4 has a simple but surprising consequence that is of interest independent from the study of degrees of randomness.

Corollary 4.1. If $X$ is n-random and $Y \leq_{T} X$ is 1-random, then $Y$ is n-random.

Proof. Immediate from parts (i) and (iv) of Theorem 3.4.

This result is relatively counterintuitive; it seems to say that it is possible to ensure a high degree of randomness by bounding the Turing degree of a 1-random real from above.

Corollary 4.1 has a parallel in the context of genericity: if $X$ is $n$-generic and $Y \leq_{T} X$ is 2-generic, then $Y$ is $n$-generic. ${ }^{2}$ The fact that it is not sufficient to assume that $Y$ is 1-generic is the subject of a recent paper of Csima, Downey, Greenberg, Hirschfeldt, and Miller [4].

Note that Nies, Stephan and Terwijn [24, Theorem 3.10] showed that a set $A$ is 2 -random iff $A$ is 1 -random and low for $\Omega$ (i.e., $\Omega$ is 1 - $A$-random). From this result - a consequence of van Lambalgen's theorem - and the fact that the low for $\Omega$ sets are obviously closed downwards under Turing reducibility, Corollary 4.1 can be concluded for $n=2$.

We wish to generalize the corollary from $n$-randomness to 1 - $Z$-randomness for an arbitrary $Z \in 2^{\omega}$. It is easy to prove this generalization if $Z$ has 1 -random Turing degree; it follows from essentially the same argument that we used in the proof of Theorem 3.4. In particular, assume that $X$ is $1-Z$-random and that $Y \leq_{T} X$ is 1-random. Furthermore, assume (without loss of generality) that $Z$ is 1-random. Then $Z$ is $1-X$-random, by van Lambalgen's theorem. So $Z$ is $1-Y$-random, which implies that $Y$ is $1-Z$-random, completing the argument.

A somewhat more complicated proof is necessary to remove the requirement that $Z$ has 1-random degree. ${ }^{3}$

Lemma 4.2. If $X \in 2^{\omega}$ is 1-random, then

$$
(\forall e)(\exists c)(\forall n) \mu\left\{A \in 2^{\omega}: \varphi_{e}^{A}\left\lceil n=X\lceil n\} \leq 2^{-n+c} .\right.\right.
$$

\footnotetext{
${ }^{2}$ We thank the referee for pointing out this result.

${ }^{3}$ The reader might hope to reduce Theorem 4.3 to the case solved above by conjecturing that if $X$ is a $1-Z$-random real, for some $Z \in 2^{\omega}$, then there is a 1-random real $\widehat{Z} \geq_{T} Z$ such that $X$ is $1-\widehat{Z}$-random. Although this would solve our problem, it is not true in general.

For a counterexample, take $X=\Omega$ and let $Z$ be any non-computable $\Delta_{2}^{0}$ low for random real; i.e., a real such that every 1-random real is $1-Z$-random. These were first constructed in [13]. By definition, $\Omega$ is 1 - $Z$-random. Now take any 1 -random real $\widehat{Z} \in 2^{\omega}$ such that $\Omega$ is 1 - $\widehat{Z}$-random; we will prove that $\widehat{Z} \Varangle_{T} Z$. By van Lambalgen's theorem, $\widehat{Z}$ is $1-\Omega$-random. But $\Omega \equiv_{T} \emptyset^{\prime}$, so $\widehat{Z}$ is 2-random. Fix $e \in \omega$ and consider the class $\mathcal{G}=\left\{A \in 2^{\omega}: \varphi_{e}^{A}=Z\right\}$. Because $Z$ is a $\Delta_{2}^{0}$ set, it is the limit of a computable sequence $\left\{Z_{s}\right\}_{s \in \omega}$ of finite sets. Thus

$$
\mathcal{G}=\left\{A \in 2^{\omega}:(\forall n)(\forall t)(\exists s \geq t) \varphi_{e, s}^{A}\left\lceil n=Z_{s}\lceil n\},\right.\right.
$$

so $\mathcal{G}$ is a $\Pi_{2}^{0}$ class. A result of Sacks [26] states that $\mu\left\{A \in 2^{\omega}: A \geq_{T} Z\right\}=0$ because $Z$ is not computable. Hence, $\mu \mathcal{G}=0$. Kurtz [14] observed that no 2-random real is contained in a measure zero $\Pi_{2}^{0}$ class, so $\varphi_{e}^{\widehat{Z}} \neq Z$. But the choice of $e$ was arbitrary, proving that $\widehat{Z} \Varangle_{T} Z$.
} 
Proof. Fix an index e. Uniformly define a family $\left\{\mathcal{H}_{\sigma}\right\}_{\sigma \in 2<\omega}$ of $\Sigma_{1}^{0}$ classes by $\mathcal{H}_{\sigma}=\left\{A \in 2^{\omega}: \varphi_{e}^{A}\lceil|\sigma|=\sigma\}\right.$. Note that if $\sigma$ and $\tau$ are incomparable strings, then $\mathcal{H}_{\sigma} \cap \mathcal{H}_{\tau}=\emptyset$. Now for each $i \in \omega$, define

$$
F_{i}=\left\{\sigma \in 2^{<\omega}: \mu \mathcal{H}_{\sigma}>2^{-|\sigma|+i}\right\} .
$$

Note that the sets $F_{i} \subseteq 2^{<\omega}$ are uniformly computably enumerable and thus $\mathcal{G}_{i}=$ $\left[F_{i}\right]$ is a uniform sequence of $\Sigma_{1}^{0}$ classes. We claim that $\mu \mathcal{G}_{i} \leq 2^{-i}$. Assume not. Then there is a prefix-free set $D \subseteq F_{i}$ such that $\mu[D]>2^{-i}$. For distinct $\sigma, \tau \in D$, we have $\mathcal{H}_{\sigma} \cap \mathcal{H}_{\tau}=\emptyset$. Therefore,

$$
\begin{aligned}
\mu\left\{A \in 2^{\omega}:(\exists \sigma \in D) \varphi_{e}^{A}\lceil|\sigma|=\sigma\}\right. & =\sum_{\sigma \in D} \mu \mathcal{H}_{\sigma} \\
>\sum_{\sigma \in D} 2^{-|\sigma|+i} & =2^{i} \sum_{\sigma \in D} 2^{-|\sigma|}=2^{i} \mu[D]>2^{i} 2^{-i}=1 .
\end{aligned}
$$

This is a contradiction, so $\mu \mathcal{G}_{i} \leq 2^{-i}$. Therefore, $\left\{\mathcal{G}_{i}\right\}_{i \in \omega}$ is a Martin-Löf test. Now let $X \in 2^{\omega}$ be 1 -random. Then $X \notin \mathcal{G}_{c}$ for some $c$. In other words,

$$
(\forall n) \mu\left\{A \in 2^{\omega}: \varphi_{e}^{A}\left\lceil n=X\lceil n\} \leq 2^{-n+c},\right.\right.
$$

which completes the proof.

Theorem 4.3. For every $Z \in 2^{\omega}$, every 1 -random real Turing reducible to a $1-Z$ random real is also $1-Z$-random.

Proof. Take $X, Y \in 2^{\omega}$ such that $X$ is 1-random and $X \leq_{T} Y$. Fix an index $e$ such that $X=\varphi_{e}^{Y}$ and let $c$ be the constant guaranteed by the previous lemma for this choice of $X$ and $e$. Now uniformly enumerate, for every $\sigma \in 2^{<\omega}$, a set of strings $F_{\sigma} \subseteq 2^{<\omega}$ as follows. Search for strings $\sigma, \tau \in 2^{<\omega}$ such that $\varphi_{e}^{\tau}\lceil|\sigma|=\sigma$ and the use of $\varphi_{e}^{\tau} \uparrow|\sigma|$ is exactly $\tau$. Whenever such strings are found, put $\tau$ into $F_{\sigma}$ provided that this maintains the condition

$$
\sum_{\tau \in F_{\sigma}} 2^{-|\tau|} \leq 2^{-|\sigma|+c}
$$

Note that each $F_{\sigma}$ is prefix-free; hence $\mu\left[F_{\sigma}\right]=\sum_{\tau \in F_{\sigma}} 2^{-|\tau|}$. Furthermore, it is clear that $\left[F_{X\lceil n}\right]=\left\{A \in 2^{\omega}: \varphi_{e}^{A}\lceil n=X\lceil n\}\right.$, for every $n$. This is because, by our choice of $c$, condition (1) does not prevent the addition of any strings to $F_{X \nmid n}$.

Now consider $Z \in 2^{\omega}$ such that $X$ is not $1-Z$-random. Our goal is to prove that $Y$ is also not $1-Z$-random. As usual, let $K^{Z}$ denote prefix-free Kolmogorov complexity relative to $Z$. For each $i \in \omega$, define a $\Sigma_{1}^{0}[Z]$ class

$$
\mathcal{G}_{i}=\bigcup_{K^{Z}(\sigma) \leq|\sigma|-c-i}\left[F_{\sigma}\right]
$$

Then

$$
\mu \mathcal{G}_{i} \leq \sum_{K^{Z}(\sigma) \leq|\sigma|-c-i} \mu\left[F_{\sigma}\right] \leq \sum_{K^{Z}(\sigma) \leq|\sigma|-c-i} 2^{-|\sigma|+c} \leq \sum_{\sigma \in 2^{<\omega}} 2^{-K^{Z}(\sigma)-i} \leq 2^{-i} .
$$

Therefore, $\left\{\mathcal{G}_{i}\right\}_{i \in \omega}$ is a Martin-Löf test relative to $Z$. Because $X$ is not 1 - $Z$-random, for each $i \in \omega$ there is an $n$ such that $K^{Z}(X\lceil n) \leq n-c-i$. But then $Y \in$ 
$\left\{A \in 2^{\omega}: \varphi_{e}^{A}\left\lceil n=X\lceil n\}=\left[F_{X\lceil n}\right] \subseteq \mathcal{G}_{i}\right.\right.$. This is true for all $i$, so $Y$ is not 1 - $Z$-random.

\section{PrefiX-Free Complexity AND the $K$-Degrees}

What properties of $X \in 2^{\omega}$ are implicit in the prefix-free complexity of its initial segments? We begin this section with a partial answer to this question; we show that the initial segment complexity of $X$ determines, for any $Z \in 2^{\omega}$, whether $X \oplus Z$ is 1 random. This proves that $X \leq_{K} Y$ implies $X \leq_{v L} Y$, so the results of the previous sections have consequences in the $K$-degrees. For example, Corollary 5.3 (i) implies that the prefix-free complexity of the initial segments of $X$ determines whether or not $X$ is $n$-random.

For the statement of the main theorem, recall that we use strings of length $n$ to represent the numbers between $2^{n}-1$ and $2^{n+1}-2$. We also need some additional notation for the proof of the theorem. Define $X \widehat{\oplus} Z$ to be

$$
\left\langle z_{0}, x_{0}, z_{1}, x_{1}, x_{2}, z_{2}, x_{3}, x_{4}, x_{5}, x_{6}, z_{3}, \ldots ., z_{n}, x_{2^{n}-1}, \ldots, x_{2^{n+1}-2}, z_{n+1}, \ldots \ldots\right\rangle,
$$

where $X=\left\langle x_{0}, x_{1}, x_{2}, \ldots\right\rangle$ and $Z=\left\langle z_{0}, z_{1}, z_{2}, \ldots\right\rangle$. It is easy to see that the class of 1-random reals is closed under computable permutations of $\omega$. Therefore, $X \widehat{\oplus} Z$ is 1-random iff $X \oplus Z$ is 1-random. In fact, $X \widehat{\oplus} Z \equiv_{v L} X \oplus Z$. We can also define $\sigma \widehat{\oplus} \tau$ for strings $\sigma, \tau \in 2^{<\omega}$, provided that $2^{|\tau|-1}-1 \leq|\sigma| \leq 2^{|\tau|}-1$.

Theorem 5.1. $X \oplus Z$ is 1-random iff $(\forall n) K(X \uparrow(Z\lceil n)) \geq Z\lceil n+n-O(1)$.

Proof. First, assume that $X \oplus Z$ is 1-random. Then $X \widehat{\oplus} Z$ is also 1-random. Note that $K(X \uparrow(Z\lceil n))=K((X \uparrow(Z\lceil n)) \widehat{\oplus}(Z\lceil n))+O(1)$ (the definition of $\widehat{\oplus}$ is contrived to ensure that $(X\lceil(Z\lceil n)) \widehat{\oplus}(Z\lceil n)$ is well defined $)$. But $(X\lceil(Z\lceil n)) \widehat{\oplus}(Z\lceil n)$ $=(X \widehat{\oplus} Z) \uparrow(Z\lceil n+n)$, so $K(X \uparrow(Z\lceil n))=K((X \widehat{\oplus} Z) \uparrow(Z\lceil n+n))+O(1) \geq$ $Z\lceil n+n-O(1)$, for all $n$.

For the other direction, define a prefix-free machine $M: 2^{<\omega} \rightarrow 2^{<\omega}$ as follows. To compute $M(\tau)$, look for $\tau_{1}, \tau_{2}, \eta_{1}$ and $\eta_{2}$ such that $\tau=\tau_{1} \tau_{2}, U\left(\tau_{1}\right)=\eta_{1} \widehat{\oplus} \eta_{2}$ and $\left|\eta_{1} \tau_{2}\right|=\eta_{2}$. If these are found, define $M(\tau)=\eta_{1} \tau_{2}$.

Assume that $X \oplus Z$ is not 1-random. Then for each $k$, there is an $m$ such that $K\left((X \widehat{\oplus} Z)\lceil m) \leq m-k\right.$. Take strings $\eta_{1}$ and $\eta_{2}$ such that $\eta_{1} \widehat{\oplus} \eta_{2}=(X \widehat{\oplus} Z)\lceil m$ and let $\tau_{1}$ be a minimal $U$-program for $\eta_{1} \widehat{\oplus} \eta_{2}$. Let $n=\left|\eta_{2}\right|$. Note that $\left|\eta_{1}\right| \leq 2^{n}-1$ and that $\eta_{2} \geq 2^{n}-1$. So, there is a string $\tau_{2}$ such that $\eta_{1} \tau_{2}=X\left\lceil\eta_{2}\right.$. Then $M\left(\tau_{1} \tau_{2}\right)=X\left\lceil\eta_{2}\right.$. Therefore,

$$
\begin{aligned}
& K\left(X \left\lceil(Z\lceil n)) \leq K\left(X\left\lceil\eta_{2}\right) \leq K_{M}\left(X\left\lceil\eta_{2}\right)+O(1) \leq\left|\tau_{1} \tau_{2}\right|+O(1)\right.\right.\right.\right. \\
& \quad \leq K\left(\eta_{1} \widehat{\oplus} \eta_{2}\right)+\left|\tau_{2}\right|+O(1) \leq\left|\eta_{1} \eta_{2}\right|-k+\left|\tau_{2}\right|+O(1) \\
& \quad=\left|\eta_{1} \tau_{2}\right|+\left|\eta_{2}\right|-k+O(1)=\eta_{2}+\left|\eta_{2}\right|-k+O(1)=Z\lceil n+n-k+O(1),
\end{aligned}
$$

where the constant depends only on $M$. Because $k$ was arbitrary, $K(X \uparrow(Z\lceil n))-$ $Z \uparrow n-n$ is not bounded below. Therefore, $(\forall n) K(X \uparrow(Z \uparrow n)) \geq Z \uparrow n+n-O(1)$ implies that $X \oplus Z$ is 1 -random.

The following corollary is immediate.

Corollary 5.2. $X \leq_{K} Y \Longrightarrow X \leq_{v L} Y$.

We see in the next section that the reverse implication fails. Combined with Theorem 3.4 and Corollaries 3.5 and 3.6, this corollary has interesting implications in the $K$-degrees. 


\section{Corollary 5.3.}

(i) If $X \leq_{K} Y$ and $X$ is n-random, then $Y$ is n-random.

(ii) If $X \oplus Y$ is 1-random, then $\left.X\right|_{K} Y$ and $X$ and $Y$ have no upper bound in the $K$-degrees. Therefore, there is no join in the $K$-degrees.

(iii) If $m \neq n$, then $\Omega^{\emptyset^{(n)}}$ and $\Omega^{\emptyset^{(m)}}$ have no upper bound in the $K$-degrees.

(iv) If $\Omega=\bigoplus_{n \in \omega} \Omega_{n}$, then $\left\{\Omega_{n}\right\}_{n \in \omega}$ is a $K$-antichain of $\Delta_{2}^{0} 1$-random reals (and again, no two have an upper bound in the $K$-degrees).

R. Rettinger has independently announced the first part of (ii): that if $X \oplus Y$ is 1-random, then $\left.X\right|_{K} Y$. Part (iii) of the corollary extends a result of $\mathrm{Yu}$, Ding and Downey (with Denis Hirschfeldt) [33]; they proved that if $p<q$, then $\Omega^{\emptyset^{(q)}} \mathbb{Z}_{K} \Omega^{\emptyset^{(p)}}$. Other connections to [33] were discussed in the introduction.

Part (i) of Corollary 5.3 implies that $n$-randomness has a characterization in terms of initial segment $K$-complexity, although not necessarily an elegant one. As an example, we give an explicit characterization of 2-randomness. By van Lambalgen's theorem $X \oplus \Omega$ is 1 -random iff $X$ is $1-\Omega$-random. But $\Omega \equiv_{T} \emptyset^{\prime}$, so $X \oplus \Omega$ is 1-random iff $X$ is 2-random. Hence, by Theorem 5.1:

Corollary 5.4. $X$ is 2-random iff $(\forall n) K(X \uparrow(\Omega \uparrow n)) \geq \Omega \uparrow n+n-O(1)$.

\section{Contrasting the $K$-Degrees And $v L$-Degrees}

The connection between the $K$-degrees and the $v L$-degrees has proved useful in understanding the $K$-degrees, but it provides only part of the picture. In this section, we prove three results that contrast $\leq_{K}$ and $\leq_{v L}$. One consequence will be that $\leq_{v L}$ does not, in general, imply $\leq_{K}$, even for $\Delta_{2}^{0} 1$-random reals. By Corollary 3.6, $\Omega$ is $v L$-below every other $\Delta_{2}^{0} 1$-random real. On the other hand, our first result implies that $\Omega \not_{K} \Omega_{0}$, where $\Omega=\Omega_{0} \oplus \Omega_{1}$. Furthermore, Proposition 6.2 gives us a $\Delta_{2}^{0} 1$-random real $X \in 2^{\omega}$ such that $X$ and $\Omega$ have no upper bound in the $K$-degrees.

Once again, recall that the string $\sigma \in 2^{<\omega}$ represents the natural number $1 \sigma-1$. So, strings of length $n$ represent numbers between $2^{n}-1$ and $2^{n+1}-2$, which implies that $\sigma \geq|\sigma|$ for every $\sigma \in 2^{<\omega}$.

Proposition 6.1. If $X \oplus Y$ is 1-random, then $\left.X\right|_{K} X \oplus Y$.

Proof. Assume that $X \oplus Y$ is 1-random. It follows from Corollary 5.2 and part (ii) of Corollary 3.5 that $X \not_{K} X \oplus Y$.

Now assume, for a contradiction, that $X \oplus Y<_{K} X$. Fix $n$ and consider $\sigma=$ $\sigma_{1} \sigma_{2} \sigma_{3} \prec X$ such that $\left|\sigma_{1}\right|=2^{n+1},\left|\sigma_{2}\right|=n$ and $\left|\sigma_{2} \sigma_{3}\right|=\sigma_{2}$. The last condition is consistent because $\sigma_{2} \geq\left|\sigma_{2}\right|$. The idea of the proof is to show that $\sigma$ has a short description, and hence $X \oplus Y \uparrow|\sigma|$ does too. But we can compute $\sigma_{2}$ from $|\sigma|$. Just take the unique number $n$ such that $2^{n+1}<|\sigma|<2^{n+2}$; then $\sigma_{2}=|\sigma|-2^{n+1}$. Thus $|\sigma|$ encodes $n$ bits of $X$ that are not in $X \oplus Y \uparrow|\sigma|$, from which we refute the randomness of $X \oplus Y$.

We turn to the details. First note that we can determine both $n$ and $|\sigma|$ from $\sigma_{2}$, so $K(\sigma) \leq K\left(\sigma_{2}\right)+\left|\sigma_{1} \sigma_{3}\right|+O(1) \leq\left|\sigma_{2}\right|+K(n)+\left|\sigma_{1} \sigma_{3}\right|+O(1) \leq|\sigma|+2 \log n+O(1)$, where the constant does not depend on $n$. Therefore, $K(X \oplus Y|| \sigma \mid) \leq|\sigma|+2 \log n+$ $O(1)$. Next we compute $K(X \oplus Y \uparrow 2|\sigma|)$. Note that $|\sigma|<2^{n+2}$, so $X \oplus Y \uparrow|\sigma|$ contains at most the first $2^{n+1}$ bits of $X$, hence no part of $\sigma_{2}$. On the other hand, $X \oplus Y \uparrow 2|\sigma|$ contains all of $\sigma_{2}$. From $X \oplus Y \uparrow|\sigma|$ we can compute $|\sigma|$, and hence $\sigma_{2}$. 
Therefore, to compute $X \oplus Y \uparrow 2|\sigma|$ from $X \oplus Y \uparrow|\sigma|$ we need to store only $|\sigma|-\left|\sigma_{2}\right|$ unknown bits. Thus $K(X \oplus Y \uparrow 2|\sigma|) \leq K(X \oplus Y \uparrow|\sigma|)+|\sigma|-\left|\sigma_{2}\right|+O(1) \leq$ $|\sigma|+2 \log n+|\sigma|-n+O(1)=2|\sigma|+2 \log n-n+O(1)$. Again, the constant does not depend on $n$. Because $n$ is arbitrary, $X \oplus Y$ is not 1-random. This contradicts our hypothesis, proving that $X \oplus Y \nless_{K} X$. Therefore, $\left.X\right|_{K} X \oplus Y$.

In light of the other results in this section, one might hope to improve Proposition 6.1 to show that $X$ and $X \oplus Y$ have no upper bound in the $K$-degrees. Surprisingly, this need not be the case. In the companion paper [22], it is proved that for every 1-random $Z \in 2^{\omega}$, there is another 1-random $X \in 2^{\omega}$ such that $X<_{K} Z$ and for every $Y \in 2^{\omega}$ we also have $X \oplus Y<_{K} Z$. By taking $Y$ to be 1- $X$-random, we get an example where $X \oplus Y$ is 1-random (by van Lambalgen's theorem) but $Z$ bounds both $X$ and $X \oplus Y$ in the $K$-degrees.

We turn to the second result of the section. A computable permutation of a 1random real is also 1-random; hence by Theorem 3.4 (iv), $v L$-degrees are invariant under computable permutations of $\omega$. On the other hand, the next proposition shows that 1-random reals $X, Y \in 2^{\omega}$ that differ only by a computable permutation can have $K$-degrees with no upper bound. In fact, we prove that there is a fixed permutation $f$ of the natural numbers such that $X$ and $f(X)$ have no $K$-upper bound for every 1-random $X \in 2^{\omega}$. This calls into question the validity of the $K$ degrees as a measure of the degree of randomness of random reals. If one believes that computably isomorphic 1-random reals should be equivalently random, then the $K$-degrees are too strong.

To understand the proof of Proposition 6.2, one should think of it as a modification of a direct proof that could have been given for Corollary 5.3 (ii): if $X$ and $Y$ are 1-random relative to each other, then they have no upper bound in the $K$ degrees. Of course, if $f: \omega \rightarrow \omega$ is a computable permutation, then $X$ and $f(X)$ are definitely not mutually 1-random, so it may not be clear how the corollary relates to the current situation. The idea is to define an $f$ that switches pairs of disjoint blocks of $\omega$ such that each pair of blocks is large enough to make the smaller blocks insignificant in comparison. Then for any 1-random real $X \in 2^{\omega}$, there are long initial segments of $X$ and $f(X)$ that behave sufficiently as if they were mutually 1random (because the vast majority of the bits are from disjoint parts of $X$ ). These initial segments are enough to prove that no real can be $K$-above both $X$ and $f(X)$.

Proposition 6.2. There is a computable permutation $f: \omega \rightarrow \omega$ such that if $X \in 2^{\omega}$ is 1-random, then $X$ and $f(X)$ have no upper bound in the $K$-degrees.

Proof. First define a computable sequence $\left\{a_{n}\right\}_{n \in \omega}$ by recursion; let $a_{0}=0$ and $a_{n+1}=a_{n}+2^{2 a_{n}+2}$, for all $n \in \omega$. Define a computable permutation of $\omega$ by

$$
f(m)= \begin{cases}m+2^{2 a_{n}+1}, & \text { if } n \in \omega \text { and } a_{n} \leq m<a_{n}+2^{2 a_{n}+1}, \\ m-2^{2 a_{n}+1}, & \text { if } n \in \omega \text { and } a_{n}+2^{2 a_{n}+1} \leq m<a_{n+1} .\end{cases}
$$

Now let $X \in 2^{\omega}$ and assume that $Z \in 2^{\omega}$ is $K$-above both $X$ and $f(X)$. We will prove that $X$ is not 1-random. Fix $n \in \omega$. Then $K\left(X \uparrow\left(a_{n}+Z \uparrow 2 a_{n}\right)\right) \leq$ $K\left(Z \uparrow\left(a_{n}+Z \uparrow 2 a_{n}\right)\right)+O(1) \leq a_{n}+Z\left\lceil 2 a_{n}+K(n)+O(1)\right.$. For the same reason, $K\left(f(X) \uparrow\left(a_{n}+Z\left\lceil 2 a_{n}\right)\right) \leq a_{n}+Z\left\lceil 2 a_{n}+K(n)+c\right.\right.$, for some $c \in \omega$ that does not depend on $n$. By Lemma 2.3 (ii), the number of strings $\tau \in 2^{a_{n}+Z \uparrow 2 a_{n}}$ such that 
$K(\tau) \leq a_{n}+Z\left\lceil 2 a_{n}+K(n)+c\right.$ is bounded by $2^{a_{n}+Z \nmid 2 a_{n}-k+O(1)}$, where

$$
\begin{aligned}
k=\left(a_{n}+Z\left\lceil 2 a_{n}+K\left(a_{n}\right.\right.\right. & \left.+Z\left\lceil 2 a_{n}\right)\right)-\left(a_{n}+Z\left\lceil 2 a_{n}+K(n)+c\right)\right. \\
& =K\left(Z\left\lceil 2 a_{n}\right)-K(n)-O(1) \geq 2 a_{n}-K(n)-O(1) .\right.
\end{aligned}
$$

Therefore, the number of such $\tau$ is bounded by $2^{Z \uparrow 2 a_{n}+K(n)-a_{n}+d}$, for some $d \in \omega$ that is again independent of $n$. We will now show that

$$
K\left(X \left\lceil\left(a_{n}+2^{2 a_{n}+1}+Z\left\lceil 2 a_{n}\right)\right) \leq a_{n}+2^{2 a_{n}+1}+Z\left\lceil 2 a_{n}-\left(a_{n}-2 K(n)\right)+O(1),\right.\right.\right.
$$

where the constant is independent of $n$. Because $\lim _{n \rightarrow \infty} a_{n}-2 K(n)=\infty$, this proves that $X$ is not 1-random.

We may assume that the universal machine $U$ was chosen so that for every $\sigma \in 2^{<\omega}$, there are $U$-programs for $\sigma$ of every length greater than $K(\sigma)$. (It suffices to define $U$ by $U\left(0^{i} 1 \sigma\right)=\widehat{U}(\sigma)$, for every $i \in \omega$ and $\sigma \in 2^{<\omega}$, where $\widehat{U}$ is an arbitrary universal machine.) So there is a $U$-program $\sigma_{1} \in 2^{a_{n}+Z \uparrow 2 a_{n}+K(n)+O(1)}$ for $X \uparrow\left(a_{n}+Z \uparrow 2 a_{n}\right)$, from which we can also determine $n, a_{n}, K(n)$ and $Z\left\lceil 2 a_{n}\right.$. Now we can effectively enumerate the strings of length $a_{n}+Z\left\lceil 2 a_{n}\right.$ with prefix-free complexity bounded by $a_{n}+Z\left\lceil 2 a_{n}+K(n)+c\right.$. Let $\sigma_{2} \in 2^{Z \uparrow 2 a_{n}+K(n)-a_{n}+d}$ code the position of $f(X) \uparrow\left(a_{n}+Z\left\lceil 2 a_{n}\right)\right.$ in this list. Given $\sigma_{1}$ and $\sigma_{2}$, since $Z\left\lceil 2 a_{n} \leq 2^{2 a_{n}+1}\right.$, we can reconstruct $a_{n}+2 \cdot Z \uparrow 2 a_{n}$ bits of $X \uparrow\left(a_{n}+2^{2 a_{n}+1}+Z \uparrow 2 a_{n}\right)$; take $\sigma_{3}$ to be the remaining bits. Finally, note that $\sigma_{1}$ is self-delimiting and that from $\sigma_{1}$ we can compute the lengths of $\sigma_{2}$ and $\sigma_{3}$. Therefore,

$$
\begin{aligned}
& K\left(X \left\lceil\left(a_{n}+2^{2 a_{n}+1}+Z\left\lceil 2 a_{n}\right)\right) \leq\left|\sigma_{1} \sigma_{2} \sigma_{3}\right|+O(1)\right.\right. \\
& =\left(a_{n}+Z\left\lceil 2 a_{n}+K(n)\right)+\left(Z\left\lceil 2 a_{n}+K(n)-a_{n}\right)+\left(2^{2 a_{n}+1}-Z\left\lceil 2 a_{n}\right)+O(1)\right.\right.\right. \\
& \quad=a_{n}+2^{2 a_{n}+1}+Z\left\lceil 2 a_{n}-\left(a_{n}-2 K(n)\right)+O(1) .\right.
\end{aligned}
$$

This completes the proof.

The final result of this section is less elegant than the previous results, but it is also more general.

Proposition 6.3. For any finite collection $X_{0}, \ldots, X_{k}$ of 1-random reals, there is another 1-random real $Y \leq_{T} X_{0} \oplus \cdots \oplus X_{k} \oplus \emptyset^{\prime}$ such that, for every $i \leq k, Y$ and $X_{i}$ have no upper bound in the $K$-degrees.

Proof. Let $\mathcal{R}=\left\{Z \in 2^{\omega}:(\forall n) K(Z\lceil n) \geq n\}\right.$ and note that $\mu \mathcal{R} \geq 1 / 2$. We define two predicates:

$$
A(\tau, p) \Longleftrightarrow \mu\{Z \succ \tau: Z \notin \mathcal{R}\}>p
$$

and $B(\sigma, s) \Longleftrightarrow(\forall i \leq k)\left(\forall \tau \in 2^{|\sigma|}\right)\left[\begin{array}{c}(\exists n<|\sigma|) K(\sigma\lceil n)>K(\tau\lceil n)+s \\ \vee(\exists n<|\sigma|) K\left(X_{i}\lceil n)>K(\tau \mid n)+s\right.\end{array}\right]$,

where $\sigma, \tau \in 2^{<\omega}, p \in \mathbb{Q}$ and $s \in \omega$. Note that if $Z$ and $X_{i}$ have no upper bound in the $K$-degrees, for every $i \leq k$, then by compactness, there is an $n$ such that $B(Z\lceil n, s)$. It should be clear that $B(\sigma, s)$ is uniformly decidable from $X_{0} \oplus \cdots \oplus X_{k} \oplus \emptyset^{\prime}$. To see that $A(\tau, p)$ can be decided by $\emptyset^{\prime}$, note that it is equivalent to $(\exists s) \mu\left\{Z \succ \tau:(\exists n \leq s) K_{s}(Z\lceil n)<n\}>p\right.$. We construct $Y=\bigcup_{s \in \omega} \sigma_{s}$ by finite initial segments $\sigma_{s} \in 2^{<\omega}$ such that $B\left(\sigma_{s+1}, s\right)$ holds. This guarantees that $X_{i}$ and $Y$ have no upper bound in the $K$-degrees, for each $i \leq k$. We also require the inductive assumption that $\mu\left(\left\{Z \in 2^{\omega}: Z \succ \sigma_{s}\right\} \cap \mathcal{R}\right)>0$. This ensures that $Y \in \mathcal{R}$ 
because $\mathcal{R}$ is closed. Therefore, $Y$ is 1-random. Finally, the construction will be done relative to the oracle $X_{0} \oplus \cdots \oplus X_{k} \oplus \emptyset^{\prime}$ to guarantee that $Y \leq_{T} X_{0} \oplus \cdots \oplus X_{k} \oplus \emptyset^{\prime}$.

Stage $s=0$ : Let $\sigma_{0}=\emptyset$. Note that $\mu\left(\left\{Z \in 2^{\omega}: Z \succ \sigma_{0}\right\} \cap \mathcal{R}\right)=\mu \mathcal{R} \geq 1 / 2>0$, so the inductive assumption holds for the base case.

Stage $s+1:$ We have constructed $\sigma_{s}$ such that $\mu\left(\left\{Z \in 2^{\omega}: Z \succ \sigma_{s}\right\} \cap \mathcal{R}\right)>0$. Using the oracle $X_{0} \oplus \cdots \oplus X_{k} \oplus \emptyset^{\prime}$, search for $\tau \succ \sigma_{s}$ and $p \in \mathbb{Q}$ such that $B(\tau, s), p<2^{-|\tau|}$ and $\neg A(\tau, p)$. If these are found, then set $\sigma_{s+1}=\tau$ and note that it satisfies our requirements. In particular, $\mu\left(\left\{Z \in 2^{\omega}: Z \succ \sigma_{s+1}\right\} \cap \mathcal{R}\right) \geq$ $2^{-\left|\sigma_{s+1}\right|}-p>0$. All that remains is to verify that the search succeeds. We know by Corollary 5.3 (ii) that if

$$
\mathcal{G}=\left\{Z \in 2^{\omega}:(\forall i \leq k) X_{i} \text { and } Z \text { have no upper bound in the } K \text {-degrees }\right\},
$$

then $\mu \mathcal{G}=1$. Therefore, $\mu\left(\mathcal{G} \cap\left[\sigma_{s}\right] \cap \mathcal{R}\right)>0$. There is a $Z \in \mathcal{G} \cap\left[\sigma_{s}\right] \cap \mathcal{R}$ such that $\mu\left([Z\lceil n] \cap \mathcal{R})>0\right.$, for all $n \in \omega$. Otherwise, $\mathcal{G} \cap\left[\sigma_{s}\right] \cap \mathcal{R}$ could be covered with a countable collection of measure zero sets. Because $Z \in \mathcal{G}$, there is an $n>\left|\sigma_{s}\right|$ such that $B\left(Z\lceil n, s)\right.$. Letting $\tau=Z\left\lceil n\right.$ ensures that $\tau \succ \sigma_{s}, B(\tau, s)$ and $\mu([\tau] \cap \mathcal{R})>0$. The last condition implies that there is a rational $p<2^{-|\tau|}$ such that $\neg A(\tau, p)$.

This completes the construction.

\section{Plain COMPleXity AND RANDOMnESS}

It turns out that much of the information implicit in the prefix-free complexity of the initial segments of a real can also be determined from the plain complexity of those initial segments. There is substance to this claim; it was not even known that 1-randomness can be characterized in terms of initial segment $C$-complexity. In Theorem 7.1 we give such characterizations. We prove that $X \in 2^{\omega}$ is 1 -random iff $(\forall n) C(X\lceil n) \geq n-K(n)-O(1)$. Although this is a natural equivalence, it falls somewhat short of giving a plain Kolmogorov complexity characterization of 1-randomness. A somewhat more satisfying solution to that problem is also provided by Theorem 7.1: $X$ is 1-random iff for every computable $g: \omega \rightarrow \omega$ such that $\sum_{n \in \omega} 2^{-g(n)}$ is finite, $(\forall n) C(X\lceil n) \geq n-g(n)-O(1)$.

Combining Theorems 5.1 and 7.1, we prove that $\leq_{C}$ implies $\leq_{v L}$. One consequence is that $n$-randomness is a $C$-degree invariant for every $n \in \omega$. As was mentioned in the introduction, 2-randomness is already known to have a $C$-complexity characterization $[21,24]: X$ is 2-random iff $\left(\exists^{\infty} n\right) C(X\lceil n) \geq n-O(1)$.

Solovay [29, section V] constructed a computable function $h: \omega \rightarrow \omega$ such that $\sum_{n \in \omega} 2^{-h(n)}<\infty$ but $\left(\exists^{\infty} n\right) h(n) \leq K(n)+O(1)$. We use a specific example of such a function in this section. Define a computable function $G: \omega \rightarrow \omega$ by

$$
G(n)=\left\{\begin{array}{ll}
K_{s+1}(t), & \text { if } n=2^{\langle s, t\rangle} \\
n, & \text { otherwise. }
\end{array} \text { and } K_{s+1}(t) \neq K_{s}(t),\right.
$$

Note that $\sum_{n \in \omega} 2^{-G(n)} \leq \sum_{n \in \omega} 2^{-n}+\sum_{t \in \omega} \sum_{m \geq K(t)} 2^{-m}=2+2 \sum_{t \in \omega} 2^{-K(t)}<$ $\infty$. We show that either $n-K(n)$ or $n-G(n)$ can be taken as the cutoff between the initial segment plain complexity of 1-random and non-1-random reals.

Theorem 7.1. For $X \in 2^{\omega}$, the following are equivalent:

(i) $X$ is 1-random.

(ii) $(\forall n) C(X\lceil n) \geq n-K(n)-O(1)$. 
(iii) $(\forall n) C(X\lceil n) \geq n-g(n)-O(1)$, for every computable $g$ : $\omega \rightarrow \omega$ such that $\sum_{n \in \omega} 2^{-g(n)}$ is finite.

(iv) $(\forall n) C(X\lceil n) \geq n-G(n)-O(1)$.

This theorem has precursors in the literature. Results closely related to (i) implies (iii) were proved by Martin-Löf [20]. Condition (ii) is similar to a characterization that Gács [9] gave of 1-randomness in terms of length conditional Kolmogorov complexity. He proved that $X \in 2^{\omega}$ is 1-random iff $(\forall n) C(X\lceil n \mid n) \geq$ $n-K(n)-O(1)$, where $C(X\lceil n \mid n)$ denotes the Kolmogorov complexity of $X\lceil n$ given $n$ (see [18] for a definition). Because $C(X\lceil n) \geq C(X\lceil n \mid n)-O(1)$, for all $X \in 2^{\omega}$ and $n \in \omega$, Gács' condition implies condition (ii) of the theorem. Therefore, the Gács characterization proves that (i) implies (ii). We give another proof of this implication below.

First, we prove the most difficult part of Theorem 7.1.

Lemma 7.2. If $(\forall n) C\left(X\lceil n) \geq n-G(n)-O(1)\right.$, then $X \in 2^{\omega}$ is 1-random.

Proof. By Lemma 2.3 (ii), there a $c \in \omega$ such that

$$
\left|\left\{\tau \in 2^{t}: K(\tau) \leq t-k\right\}\right| \leq 2^{t-K(t)-k+c},
$$

for all $t, k \in \omega$. We construct a partial computable (non-prefix-free) function $M: 2^{<\omega} \rightarrow 2^{<\omega}$. For $s, t \in \omega$, let $n=2^{\langle s, t\rangle}$. To $\langle s, t\rangle$ we devote the $M$-programs with lengths from $n / 2+c+1$ to $n+c$. Note that distinct pairs do not compete for elements in the domain of $M$. For $k \in \omega$, let $m=n-K_{s+1}(t)-k+c$. Clearly, $m \leq n+c$. If $m \geq n / 2+c+1$, then for every $\sigma \in 2^{n}$ such that $K(\sigma\lceil t) \leq t-k$, try to give $\sigma$ an $M$-program of length $m$. Different $k$ do not compete for programs, but it is still possible that there are not enough strings of length $m$ for all such $\sigma$. However, this cannot happen if $K_{s}(t)=K(t)$. This is because the number of $\sigma \in 2^{n}$ for which $K\left(\sigma\lceil t) \leq t-k\right.$ is bounded above by $2^{t-K(t)-k+c} 2^{n-t}=2^{n-K(t)-k+c}=2^{m}$, so there is enough room in the domain of $M$ to handle every such $\sigma$. This completes the construction of $M$.

Assume that $X \in 2^{\omega}$ is not 1-random. For each $k \in \omega$, there is a $t \in \omega$ such that $K\left(X\lceil t) \leq t-k\right.$ and $t$ is large enough that $K(t) \leq 2^{t-1}-k-1$. Take the least $s \in \omega$ such that $K_{s+1}(t)=K(t)$ and let $n=2^{\langle s, t\rangle}$. Then

$$
m=n-K(t)-k+c \geq n-2^{t-1}+k+1-k-c \geq n / 2+c+1,
$$

because $n=2^{\langle s, t\rangle} \geq 2^{t}$. This implies that there is an $M$-program for $X\lceil n$ of length $m=n-K(t)-k+c$. Also note that $G(n)=K_{s+1}(t)=K(t)$. So,

$$
C\left(X\lceil n) \leq C_{M}(X\lceil n)+O(1) \leq n-K(t)-k+c+O(1) \leq n-G(n)-k+O(1),\right.
$$

where the constant is independent of $X, n$ and $k$. Because $k$ is arbitrary,

$$
\liminf _{n \rightarrow \infty} C(X\lceil n)-n+G(n)=-\infty .
$$

Therefore, if $(\forall n) C(X\lceil n) \geq n-G(n)-O(1)$, then $X$ is 1-random. This completes the proof.

Proof of Theorem 7.1. (i) $\Longrightarrow$ (ii): Define

$$
\mathcal{I}_{k}=\left\{X \in 2^{\omega}:(\exists n) C(X\lceil n)<n-K(n)-k\} .\right.
$$

As usual, let $K_{s}$ and $C_{s}$ denote the approximations to $K$ and $C$ at stage $s$. Then $(\exists n)(\exists s) C_{s}\left(X\lceil n)+K_{s}(n)<n-k\right.$ iff $X \in \mathcal{I}_{k}$. Therefore, $\mathcal{I}_{k}$ is a $\Sigma_{1}^{0}$ class. 
Fewer than $2^{n-K(n)-k} V$-programs have length less than $n-K(n)-k$, so $\mid\{\sigma \in$ $\left.2^{n}: C(\sigma)<n-K(n)-k\right\} \mid \leq 2^{n-K(n)-k}$. Therefore,

$$
\begin{aligned}
\mu \mathcal{I}_{k} \leq \sum_{n \in \omega} \mu\left\{X \in 2^{\omega}: C(X\lceil n)\right. & <n-K(n)-k\} \\
& \leq \sum_{n \in \omega} 2^{-n} 2^{n-K(n)-k}=2^{-k} \sum_{n \in \omega} 2^{-K(n)} \leq 2^{-k} .
\end{aligned}
$$

So, $\left\{\mathcal{I}_{k}\right\}_{k \in \omega}$ is a Martin-Löf test. If $X$ is 1-random, then $X \notin \mathcal{I}_{k}$ for large enough $k$. In other words, $(\forall n) C(X\lceil n) \geq n-K(n)-k$.

(ii) $\Longrightarrow$ (iii): Let $g: \omega \rightarrow \omega$ be a computable function such that $\sum_{n \in \omega} 2^{-g(n)}<$ $\infty$. By the minimality of $K$ as an information content measure, $(\forall n) K(n) \leq$ $g(n)+O(1)$. Therefore, if $(\forall n) C(X\lceil n) \geq n-K(n)-O(1)$, then $(\forall n) C(X\lceil n) \geq$ $n-g(n)-O(1)$.

(iii) $\Longrightarrow$ (iv) is immediate because $G$ is computable and $\sum_{n \in \omega} 2^{-G(n)}$ is finite.

Finally, (iv) $\Longrightarrow$ (i) was proved in Lemma 7.2.

As with $K$-complexity, the $C$-complexity of the initial segments of a real determines its $v L$-degree. This is a consequence of the following result.

Theorem 7.3. Assume that $Z \in 2^{\omega}$ is 1-random. The following are equivalent:

(i) $X$ is 1-Z-random.

(ii) $(\forall n) C\left(X\lceil n) \geq n-K^{Z}(n)-O(1)\right.$.

(iii) $(\forall n) C(X\lceil n)+K(Z\lceil n) \geq 2 n-O(1)$.

The following lemma is folklore (see [18, page 138]).

Lemma 7.4. For any real $X \in 2^{\omega},(\forall n) C(X\lceil(X\lceil n)) \leq X\lceil n-n+O(1)$.

Proof of Theorem 7.3. (i) $\Longrightarrow$ (ii): Suppose that $Z$ is 1-random. If $X$ is $1-Z$ random, then by relativizing Theorem 7.1,

$$
(\forall n) C\left(X\lceil n) \geq C^{Z}\left(X\lceil n)-O(1) \geq n-K^{Z}(n)-O(1) .\right.\right.
$$

(ii) $\Longrightarrow$ (iii): Since $Z$ is 1-random, the ample excess lemma gives $K^{Z}(n) \leq$ $K\left(Z\lceil n)-n+O(1)\right.$, for all $n \in \omega$. So $(\forall n) C\left(X\lceil n) \geq n-K^{Z}(n)-O(1) \geq\right.$ $2 n-K(Z\lceil n)-O(1)$.

(iii) $\Longrightarrow$ (i): By Lemma 7.4, $(\forall n) K(Z \uparrow(X\lceil n)) \geq 2 \cdot X\lceil n-C(X \uparrow(X\lceil n))-$ $O(1) \geq 2 \cdot X\lceil n-X\lceil n+n-O(1) \geq X\lceil n+n-O(1)$. By Theorem 5.1, $Z \oplus X$ is 1 -random. So, by Theorem 3.1, $X$ is 1 - $Z$-random.

Note that assuming (iii), we have $K(Z\lceil n) \geq 2 n-C(X\lceil n)-O(1) \geq n-O(1)$ for all $n \in \omega$, so $Z$ is 1 -random. This gives us a cleaner way of expressing the equivalence of (i) and (iii).

Corollary 7.5. $X \oplus Z$ is 1-random iff $(\forall n) C(X\lceil n)+K(Z\lceil n) \geq 2 n-O(1)$.

An immediate consequence is that the $C$-degrees refine the $v L$-degrees.

Corollary 7.6. $X \leq_{C} Y \Longrightarrow X \leq_{v L} Y$.

Therefore, the conclusions of Corollary 5.3 hold for the $C$-degrees as well. 


\section{Corollary 7.7.}

(i) If $X \leq_{C} Y$ and $X$ is n-random, then $Y$ is n-random.

(ii) If $X \oplus Y$ is 1-random, then $\left.X\right|_{C} Y$ and $X$ and $Y$ have no upper bound in the $C$-degrees. Therefore, there is no join in the $C$-degrees.

(iii) If $m \neq n$, then $\Omega^{\emptyset^{(n)}}$ and $\Omega^{\emptyset^{(m)}}$ have no upper bound in the $C$-degrees.

(iv) If $\Omega=\bigoplus_{n \in \omega} \Omega_{n}$, then $\left\{\Omega_{n}\right\}_{n \in \omega}$ is a $C$-antichain of $\Delta_{2}^{0} 1$-random reals (and again, no two have an upper bound in the $C$-degrees).

Finally, we remark that it requires only superficial modification to the proof of Proposition 6.3 to prove the corresponding result for the $C$-degrees: if $X_{0}, \ldots, X_{k}$ are 1-random reals, then there is a 1-random real $Y \leq_{T} X_{0} \oplus \cdots \oplus X_{k} \oplus \emptyset^{\prime}$ such that, for every $i \leq k, Y$ and $X_{i}$ have no upper bound in the $C$-degrees. This implies that there is a $\Delta_{2}^{0} 1$-random real that is not $C$-above $\Omega$; hence $\leq_{v L}$ and $\leq_{C}$ differ on the 1-random reals.

\section{REFERENCES}

1. Gregory J. Chaitin, On the length of programs for computing finite binary sequences: Statistical considerations, J. Assoc. Comput. Mach. 16 (1969), 145-159. MR0237121 (38:5414)

2. A theory of program size formally identical to information theory, J. Assoc. Comput. Mach. 22 (1975), 329-340. MR0411829 (53:15557)

3. Incompleteness theorems for random reals, Adv. in Appl. Math. 8 (1987), no. 2, 119-146. MR886921 (88h:68038)

4. Barbara F. Csima, Rod G. Downey, Noam Greenberg, Denis R. Hirschfeldt, and Joseph S. Miller, Every 1-generic computes a properly 1-generic, Journal of Symbolic Logic. 71 (2006), 1385-1393. MR2275865 (2007h:03083)

5. R. Downey and D. Hirschfeldt, Algorithmic randomness and complexity, Springer-Verlag, Berlin, To appear.

6. Rod G. Downey, Denis R. Hirschfeldt, and Geoff LaForte, Randomness and reducibility (extended abstract), Mathematical foundations of computer science, 2001 (Mariánské Láznĕ), Lecture Notes in Comput. Sci., vol. 2136, Springer, Berlin, 2001, pp. 316-327. MR1907022 (2003j:03051)

7. [6] for an extended abstract. MR2030512 (2004m:03165)

8. Rod G. Downey, Denis R. Hirschfeldt, Joseph S. Miller, and André Nies, Relativizing Chaitin's halting probability, J. Math. Log. 5 (2005), no. 2, 167-192. MR2188515 (2007e:68031)

9. Péter Gács, Exact expressions for some randomness tests, Z. Math. Logik Grundlag. Math. 26 (1980), no. 5, 385-394. MR82c:65004

10. Every sequence is reducible to a random one, Inform. and Control 70 (1986), no. 2-3, 186-192. MR87k:03043

11. A. N. Kolmogorov, Three approaches to the quantitative definition of information, Internat. J. Comput. Math. 2 (1968), 157-168. MR0243922 (39:5240)

12. Antonín Kučera, Measure, $\Pi_{1}^{0}$-classes and complete extensions of PA, Recursion theory week (Oberwolfach, 1984), Lecture Notes in Math., vol. 1141, Springer, Berlin, 1985, pp. 245-259. MR87e:03102

13. Antonín Kučera and Sebastiaan A. Terwijn, Lowness for the class of random sets, J. Symbolic Logic 64 (1999), no. 4, 1396-1402. MR2001j:03082

14. S. Kurtz, Randomness and genericity in the degrees of unsolvability, Ph.D. Dissertation, University of Illinois, Urbana, 1981.

15. Manuel Lerman, Degrees of unsolvability, Perspectives in Mathematical Logic, SpringerVerlag, Berlin, 1983, Local and global theory. MR85h:03044

16. L. A. Levin, Laws of information conservation (non-growth) and aspects of the foundation of probability theory, Problems Inform. Transmission 10 (1974), no. 3, 206-210. MR0469513 $(57: 9298)$

17. MR366096 (51:2346) 
18. M. Li and P. Vitányi, An introduction to Kolmogorov complexity and its applications, Texts and Monographs in Computer Science, Springer-Verlag, New York, 1993. MR94j:68121

19. Per Martin-Löf, The definition of random sequences, Information and Control 9 (1966), 602619. MR0223179 (36:6228)

20. Complexity oscillations in infinite binary sequences, Z. Wahrscheinlichkeitstheorie und Verw. Gebiete 19 (1971), 225-230. MR0451322 (56:9609)

21. Joseph S. Miller, Every 2-random real is Kolmogorov random, J. Symbolic Logic 69 (2004), no. 3, 907-913. MR2078929 (2005e:68108)

22. Joseph S. Miller and Liang Yu, Oscillation in the initial segment complexity of random reals, In preparation.

23. André Nies, Lowness properties and randomness, Adv. Math. 197 (2005), no. 1, 274-305. MR2166184

24. André Nies, Frank Stephan, and Sebastiaan A. Terwijn, Randomness, relativization and Turing degrees, J. Symbolic Logic 70 (2005), no. 2, 515-535. MR2140044

25. John C. Oxtoby, Measure and category, second ed., Graduate Texts in Mathematics, vol. 2, Springer-Verlag, New York, 1980, A survey of the analogies between topological and measure spaces. MR81j:28003

26. Gerald E. Sacks, Degrees of unsolvability, Princeton University Press, Princeton, N.J., 1963. MR32:4013

27. Robert I. Soare, Recursively enumerable sets and degrees, Springer-Verlag, Berlin, 1987. MR88m:03003

28. R. J. Solomonoff, A formal theory of inductive inference, Information and Control 7 (1964), 1-22, 224-254.

29. Robert M. Solovay, Draft of paper (or series of papers) on Chaitin's work, (1975), Unpublished notes, 215 pages.

30. M. van Lambalgen, Random sequences, Ph.D. Dissertation, University of Amsterdam, 1987.

31. Michiel van Lambalgen, The axiomatization of randomness, J. Symbolic Logic 55 (1990), no. 3, 1143-1167. MR92f:68075

32. Liang $\mathrm{Yu}$ and Decheng Ding, There are $2^{\aleph_{0}}$ many $H$-degrees in the random reals, Proc. Amer. Math. Soc. 132 (2004), no. 8, 2461-2464 (electronic). MR2052426 (2004m:68109)

33. Liang Yu, Decheng Ding, and Rod G. Downey, The Kolmogorov complexity of random reals, Ann. Pure Appl. Logic 129 (2004), no. 1-3, 163-180. MR2078364 (2005e:68109)

34. A. K. Zvonkin and L. A. Levin, The complexity of finite objects and the development of the concepts of information and randomness by means of the theory of algorithms, Russian Math. Surveys 25 (1970), no. 6, 83-124. MR0307889 (46:7004)

Department of Mathematics, University of Connecticut, Storrs, Connecticut 062693009

E-mail address: joseph.miller@math.uconn.edu

Department of Mathematics, National University of Singapore, Singapore 117543

E-mail address: yuliang.nju@gmail.com 